\title{
Methods of introduction of MgO nanoparticles into Bi-2212/Ag tapes
}

Igor E. Agranovski ${ }^{\mathrm{a}, *}$, Alexander Y. Ilyushechkin ${ }^{\mathrm{b}}$, Igor S. Altman ${ }^{\mathrm{a}, \mathrm{c}}$, Thor E. Bostrom ${ }^{\mathrm{d}}$, Mansoo Choi ${ }^{\mathrm{c}}$

${ }^{a}$ Faculty of Environmental Sciences, Griffith University, Brisbane, Qld 4111, Australia

${ }^{b}$ CSIRO, Pullenvale, 4069, QLD, Australia

${ }^{\mathrm{c}}$ National CRI Center for Nano Particle Control, Institute of Advanced Machinery and Design, Seoul National University, Seoul 151-742, Republic of Korea

${ }^{\mathrm{d}}$ School of Physical and Chemical Sciences, Queensland University of Technology, P.O. Box 2434, Brisbane, Qld 4001, Australia

Submitted to:

Physica C

5 October 2005

Published:

Physica C 434: 115-120 (2006)

* Corresponding author. Tel.: +61 73875 7923; fax: +61 738757459 .

E-mail address: i.agranovski@griffith.edu.au (I.E. Agranovski) 


\begin{abstract}
The effect of addition of ultra-fine MgO particles, obtained by Mg combustion, in Bi2212/Ag tapes was investigated. Bi-2212/Ag tapes were prepared by coating and partial melt processing. Four different methods of MgO particles embedment were applied in green tape preparation; settlement of $\mathrm{MgO}$ in $\mathrm{Bi}-2212$, pre-mixing of $\mathrm{MgO}$ in the solvent, deposition of the MgO into the solvent, and deposition of MgO onto the surface of Bi-2212. Microstructural studies of processed tapes show that uniformity of $\mathrm{MgO}$ particles distribution in $\mathrm{Bi}-2212$ matrix depends on the methods of tape preparation.
\end{abstract}

MgO particles, introduced in Bi-2212 matrix by all applied methods, significantly enhanced transport properties of the tapes. The $J_{c}$ degradation with the increase of magnetic field and/or temperature in all doped samples was significantly lower compared to un-doped Bi-2212 samples. The best improvement was achieved when $\mathrm{MgO}$ particles were introduced into $\mathrm{Bi}-2212$ matrix by settlement method of the tape coating. The improvement also depends on a magnitude of the magnetic field and operating temperatures.

\title{
1. Introduction
}

$\mathrm{Bi}_{2} \mathrm{Sr}_{2} \mathrm{CaCu}_{2} \mathrm{Oy}(\mathrm{Bi}-2212)$ high temperature superconductor is one of the good candidates for the power application, because of the high critical current density at low temperatures and high magnetic fields and relatively easy fabrication $[1,2]$. However, critical current densities of $\mathrm{Bi}-2212$ bulk and tapes decrease with increasing 
temperature and applied field due to weak flux pinning. The current density can be enhanced by the addition of pinning centers $[3,4]$. One of the possible solutions to introduce flux-pinning centers in superconductors is by an addition of MgO particles, which are incorporated into the $\mathrm{Bi}-2212$ matrix [4,5]. It was also found that $\mathrm{MgO}$ addition into $\mathrm{Bi}-2212$ bulk can improve texturing [6], and reduce amount of the secondary phases in Bi-2212 thick films [4].

The improvement of the critical current densities of $\mathrm{Bi}-2212 / \mathrm{Ni}$ and $\mathrm{Bi}-2212$ /Ag composite tapes has been demonstrated with the addition of MgO nanoparticles [7-10]. The particle size of MgO may play a significant role in the efficiency of the flux pinning centers. If the size of particles is much greater than the coherence length of high-temperature superconductors, it is believed [4] that the extra pinning centers must be the interface between the MgO particles and the superconductor matrix.

In our previous work, we investigated the effect of the addition of ultra-fine MgO particles on the microstructure and superconducting properties of $\mathrm{Bi}-2212 / \mathrm{Ag}$ tapes, prepared by dip-coating and partial melting process [11]. Ultra-fine MgO particles with cubic morphology and average particle size of 40nm were obtained by burning out a single Mg particle in air as described elsewhere [12].

It was found that the significant enhancement in magnetization hysteresis below the transition temperature could be achieved only if MgO ultra-fine particles are individually dispersed in the $\mathrm{Bi}-2212$ matrix [11]. In order to prevent $\mathrm{MgO}$ particle agglomeration in the $\mathrm{Bi}-2212 / \mathrm{Ag}$ tapes, an alternative method of $\mathrm{MgO}$ particle introduction into the Bi-2212 matrix was applied, namely the direct deposition of $\mathrm{MgO}$ during the $\mathrm{Mg}$ particle combustion; and the influence of the distribution of the $\mathrm{MgO}$ particles on the transport properties of B-2212/Ag tapes was investigated. However, the above method does not provide a uniform distribution of 
the MgO concentration across the tape thickness. It allows one to speculate that the MgO doping effect on the critical current density of Bi-2212 tapes could be improved further if the MgO concentration could be made constant in the $\mathrm{Bi}-2212$ matrix.

The focus of the present work is to develop methods for the introduction of MgO into a $\mathrm{Bi}-2212$ matrix in order to obtain uniform distribution of the ultra-fine particles in the Bi-2212 matrix and conserve their appearance as individual particles.

\section{Methods}

\section{1. $\mathrm{MgO}$ particle preparation}

The MgO nanosize particles were produced by burning a single Mg particle in the air. A $3 \mathrm{~mm} \mathrm{Mg}$ piece supported by a $250-\mu \mathrm{m}$ tungsten wire was ignited by a small propane-air diffusion flame, which was removed immediately after the commencement of the metal combustion. Due to natural convection, the $\mathrm{MgO}$ nanoparticles formed in the generation zone surrounding the $\mathrm{Mg}$ particle travel upward. The MgO fume was collected either onto a glass plate or directly onto a Bi2212 surface held about $5 \mathrm{~mm}$ above the burning Mg.

\subsection{Green tape preparation}

Four different methods have been developed to introduce MgO particles into the $\mathrm{Bi}-2212$ matrix.

\subsubsection{Method 1: settlement}

Freshly produced MgO nanoparticles (see Section 2.1) were collected on the glass substrate, carefully brushed from it and mixed with the pure ethyl alcohol $\left(\mathrm{C}_{2} \mathrm{H}_{5} \mathrm{OH}\right)$ with the following treatment for $15 \mathrm{~min}$ in an ultrasonic bath to break possible agglomerates. Bi-2212 powder, previously sieved through 325 mesh, was added into the $\mathrm{MgO} / \mathrm{C}_{2} \mathrm{H}_{5} \mathrm{OH}$ suspension and the new mixture was sonicated for 
another 15 minutes. A $3 \mathrm{~mm}$ wide and $30 \mathrm{~mm}$ long silver strip was placed at the bottom of the slot specially designed to accommodate Ag tapes. Then the suspension was added into the slot by analytical pipette and left until full evaporation of the alcohol and corresponding settlement of the powder mixture on the strip surface. The strip was then carefully removed from the slot and placed into the furnace for heat treatment. The thickness of the film was controlled by the amount of suspension added into the slot.

\subsubsection{Method 2: pre-mixing of MgO nanoparticles in the solvent}

Freshly made $\mathrm{MgO}$ nanoparticles were firstly mixed with the solvent to ensure their best possible spread. The sieved $\mathrm{Bi}-2212$ powder was then added to the $\mathrm{MgO} /$ solvent and mixed for at least $10 \mathrm{~min}$. Such a method alters from the standard procedure [11] where the dry MgO was firstly mixed with the dry Bi-2212 with the following addition of the solvent, as the effectiveness of deagglomeration of MgO in the pure solvent is much higher compared to the case of presence of $\mathrm{Bi}-2212$ in it. The green tapes were then prepared by a dip-coating process [11] and heat treated in the furnace to produce the final superconducting tape.

\subsubsection{Method 3: direct deposition of $\mathrm{MgO}$ particles into the solvent}

To prepare the paste by this method, solvent was firstly spread along a silicon substrate to create a thin film covering the surface to be used for collection of MgO nanoparticles during $\mathrm{Mg}$ combustion. The $\mathrm{Mg}$ particle was ignited and $\mathrm{MgO}$ nanoparticles were collected directly into the solvent film. After completion of collection, the solvent film containing loose MgO particles was gently wiped from the surface and used for mixing with the Bi-2212 powder for the subsequent partial melting procedure performed in the furnace. The concentration of $\mathrm{MgO}$ was controlled by the following procedure: a silicon substrate with the solvent film of 
known weight was placed in the nanoparticle stream for a certain measured time period. After particle collection, the film was gently wiped and thoroughly mixed. A droplet of the $\mathrm{MgO} /$ solvent mixture was then placed onto the surface of the $10 \times 10$ mm pieces of glass previously weighed on the analytical balance. After placement of the droplet, the glass piece was weighed again and located in the oven for treatment at $500^{\circ} \mathrm{C}$ for $2 \mathrm{~h}$. On completion of this procedure (ensuring that the whole amount of solvent was removed from the glass), the glass piece was weighed again to identify the amount of $\mathrm{MgO}$ remaining on the surface. As the result of this procedure, the time related concentration of $\mathrm{MgO}$ in the solvent was obtained and used as a scale factor for the rest of experiments.

2.2.4. Method 4: deposition of MgO nanoparticles onto the surface of Bi-2212

The last method of green tape preparation was based on the deposition of MgO nanoparticles onto the dry surface of the Bi-2212 previously produced on the silver tape. The detailed description of this method is provided in our previous publication [11] and those green tapes were used for comparison with tapes produced by the other methods described above.

\subsection{Tapes heat-treatment}

Green tapes (single or laminated) of $30 \mathrm{~mm}$ length were placed on a ceramic support for heat treatment using a partial melt method in flowing oxygen [13]. Using differential thermal analysis (DTA) measurements, partial melting temperatures, $T_{\max }$, between 890 and $900{ }^{\circ} \mathrm{C}$ were chosen to find the optimum processing temperature for different green film compositions.

After heat treatment, tapes were annealed in an inert atmosphere to optimise oxygen content in Bi-2212 matrix. It was found that oxygen content in Bi-2212 thick 
films, corresponded to highest critical current densities, can be achieved by postannealing in nitrogen atmosphere $\left(\mathrm{pO}_{2} \sim 0.01 \mathrm{~atm}\right)$ at $500{ }^{\circ} \mathrm{C}$ for $20 \mathrm{~h}$ [14]. Similar post-annealing procedure was optimized in our previous studies [11,13]; it was determined that for pure Bi-2212/Ag and MgO-doped laminated tapes, post-annealing in flowing argon $\left(\mathrm{pO}_{2} \sim 0.01 \mathrm{~atm}\right)$ at $600{ }^{\circ} \mathrm{C}$ for $10 \mathrm{~h}$ provides highest critical current densities in the tapes.

\subsection{Sample characterization}

The microstructure, composition and thickness of samples were determined using a scanning electron microscope (FEI Quanta 200 SEM) equipped with an EDAX energy-dispersive X-ray spectrometry (EDX) system. SEM imaging was usually carried out using a backscattered electron detector to distinguish the $\mathrm{MgO}$ from the much higher atomic number matrix.

Critical current measurements were carried out at $77 \mathrm{~K}$ in self-field using a standard four probe method with $1 \mu \mathrm{V} \mathrm{cm}{ }^{-1}$ criterion. In these measurements, current and voltage electrodes were attached using a soldering iron. The voltage contacts were placed over the samples at distances of about $10 \mathrm{~mm}$ from each other.

The magnetization critical current density was obtained from magnetization hysteresis curves, using the Bean model of the critical state [15]. The magnetic characteristics of the samples were measured with a commercial SQUID (Superconducting Quantum Interference Device) magnetometer (Quantum Design, MPMS 7) at temperatures down to $5 \mathrm{~K}$. In measurements, the magnetic field was applied in parallel to the c-axis of the Bi-2212/Ag tapes. The relative error of the measured magnetization typically did not exceed $1 \%$. 
A concentration distribution of the $\mathrm{MgO}$ along the $c$-axis in the tapes was determined by quantitative EDX analysis (see [11] for details). Every $5 \mu \mathrm{m}$ of the tape's cross section was scanned in the direction of the $c$-axis. The net X-ray peak intensities were then normalized to the value, obtained from the overall tape cross section, which corresponded to the initial concentration of MgO in the tapes.

\section{Results and discussion}

\subsection{Summary of methods used for $\mathrm{MgO}$ particle introduction into the Bi-}

\section{2 matrix}

Each method used for particle introduction has its own benefits and "bottlenecks". The settlement method is the least labor consuming and provides the most uniform MgO distribution across the Bi-2212 matrix. However, the film produced after settlement is very fragile with the tapes and, especially, laminated tapes require very careful handling during transportation to the heat treatment stage. Such a problem is fully addressed in both methods utilizing the addition of the MgO particles to the solvent with subsequent mixing with the Bi-2212 powder. However the distribution of MgO particles measured for these methods is not as uniform as that for the settlement method. Moreover, for the method based on the direct deposition of the $\mathrm{MgO}$ particles into the solvent, the concentration of $\mathrm{MgO}$ particles could be controlled much less precisely than for the other methods used. The other issue relating to this method is the possible alteration of physical properties of the solvent after direct deposition of the hot MgO particles into it; such alterations may not be beneficial for the tape performance. Finally, the main issues relating to the tapes produced by method 4 are discussed in detail in [11]. 


\subsection{Microstructure of the processed Bi-2212/Ag tapes and $\mathrm{MgO}$}

\section{distribution}

SEM observation revealed that partially melted tapes consisted of the Bi-2212 matrix as a major phase together with some secondary phases, which were identified by EDX as a Bi-free and a minor $\mathrm{Cu}$-free phase. The amount and size of the Bi-free phase in the tapes with $\mathrm{MgO}$ addition was significantly lower compared to the undoped Bi-2212 tapes.

Typical surfaces of tapes, prepared by different methods of MgO introduction, are shown in Fig. 1. In all methods, the MgO does not react with the Bi-2212 matrix and appears as individual (loose) particles which retain their original cubic shape. Such a distribution was already observed in the tapes where the MgO was introduced by deposition [11] onto the surface of previously produced Bi-2212. All SEM images correspond to the samples containing 4 wt.\% of the MgO. A relatively high concentration of the $\mathrm{MgO}$ on the surface of the samples obtained by the "MgO deposition” method is expected because all $\mathrm{MgO}$ particles were initially deposited on the surface (Fig. 1a). Also, as was shown and discussed before [11], after partial melting, the MgO particle concentration on the surface remains higher than in the overall cross-section of these tapes.

The overall uniformity of the MgO particle distribution across the tape was found to vary with the preparation methods used. The most homogeneous MgO distribution was obtained for the tapes prepared by the settlement method (Fig. 1b). In addition, the EDX results obtained for these tapes show that the variation of the MgO concentration along the $c$-axis did not exceed $10 \%$. 
A satisfactory MgO distribution was achieved for the case when deposited MgO particles were mixed with organic solvent (Fig. 1c) with the corresponding variation of the $\mathrm{MgO}$ concentration across the $c$-axis not exceeding 15\%, as measured by EDX.

Some local concentrations of the MgO particles were observed in the samples prepared by the solvent deposition method. In the samples which exhibited these concentrations, it was also observed that a Cu-free phase was present in the surrounding of MgO-free areas (Fig. 1d). The EDX results were, nevertheless, satisfactory with the MgO concentration variation within 25\%.

\subsection{Transport properties of the tapes in magnetic field}

Based on the DTA results, the processing parameters for the tapes were chosen in order to obtain the highest value of the critical current density at $77 \mathrm{~K}$ and self-field. Maximum melting temperature is one of the important parameters, which affects $\mathrm{Bi}-2212$ structure and, therefore, superconducting properties [14]. In the previous study [11], it was found that highest critical current densities in the Bi-2212 $+\mathrm{MgO}$ tapes can be achieved when the partial melting temperature is $892-897{ }^{\circ} \mathrm{C}$, which is about $10-20{ }^{\circ} \mathrm{C}$ higher then the temperature of the $\mathrm{Bi}-2212$ melting point. The other processing parameters, such as cooling rates and annealing time, have been previously optimized for pure Bi-2212 composition [13]. As was found in this study, the $\mathrm{MgO}$ addition into the $\mathrm{Bi}-2212$ does not change these parameters in order to obtained maximum $J_{\mathrm{c}}$.

In present work, $J_{\mathrm{c}}$ of the partially melted tapes vary from 2,500 to 15,000 $\mathrm{A} / \mathrm{cm}^{2}$ at $77 \mathrm{~K}$ and zero field, depending on the tapes thickness and $\mathrm{MgO}$ concentration. Fig. 2 shows $J_{\mathrm{c}}$-thickness dependence for undoped and MgO-doped Bi- 
2212 tapes. It is clear, that $J_{\mathrm{c}}$ becomes significantly lower when the thickness of the films increases, particularly when films are 30-40 $\mu \mathrm{m}$ and thinner. The results obtained by Buhl et al. [14] for Bi-2212 thick films on Ag substrate show exactly the same trend.

The results on the optimization of the $\mathrm{MgO}$ concentration will be presented separately. Generally, the highest $J_{\mathrm{c}}$ values were achieved in the Bi-2212 tapes with 4 wt. \% of MgO for all methods, except “MgO deposition”, which produced tapes with a high gradient of the concentration along the $c$-axis of the tape. Tapes prepared by settlement and solvent mixed methods have similar $J_{\mathrm{c}}$ values, while $J_{\mathrm{c}}$ 's of the solvent-deposited tapes are relatively lower. This can be explained by local nonhomogeneities of the MgO distribution, which may partially block current paths.

Since $J_{\mathrm{c}}$ of the Bi-2212 tapes depends on the thickness of the Bi-2212 ceramic layer, to evaluate the effect of MgO additive applied by different methods it is preferable to have the tapes with similar thicknesses or normalized $J_{\mathrm{c}}$ values. Absolute $J_{\mathrm{c}}$ values are also dependent on the secondary phase content, grain misalignment and other defects.

In this work we present the results of investigation of the $J_{\mathrm{c}}$ behavior of the Bi$2212+\mathrm{MgO} / \mathrm{Ag}$ tapes in the applied magnetic field at a range of temperatures. The $J_{\mathrm{c}}$ values were normalized to the values obtained at $0.1 \mathrm{~T}$ field (for field dependence) or $5 \mathrm{~K}$ (for temperature dependence).

Fig. 3a and b shows the effect of the magnetic field on the normalized $J_{\mathrm{c}}$ at temperatures of $5 \mathrm{~K}$ and $20 \mathrm{~K}$, respectively. The graphs represent the results of measurements of the tapes produced by four different methods of $\mathrm{MgO}$ introduction along with the results obtained for the un-doped Bi-2212/Ag tape plotted for comparison. All samples show degradation with increase of the field, however MgO- 
doped samples degrade less compared to the un-doped ones. Also, as is seen, at the temperature of $5 \mathrm{~K}$, samples obtained by settlement, solvent mixed and solvent deposit methods show the best results; their normalized $J_{\text {c }}$ 's are about 1.5 times higher compared to the results obtained for the un-doped samples. With the temperature increase to $20 \mathrm{~K}$ this difference becomes even more significant. In addition, for the field region between 2 and $4 \mathrm{~T}$, both samples from the solvents methods have 5-8 times higher $J_{\mathrm{C}}$ 's, whilst the samples produced by the settlement method have $J_{\mathrm{c}}$ 's more than an order of magnitude higher compared to the un-doped samples.

The $J_{\mathrm{c}}-\mathrm{T}$ curves are shown in Fig. $4 \mathrm{a}$ and $\mathrm{b}$ at magnetic fields of 0.1 and $1 \mathrm{~T}$, respectively. At low fields, the $J_{\mathrm{c}}$ performance of the tape with deposited MgO particles improved with temperature increasing over those of the tape without $\mathrm{MgO}$ by a factor of 2.5-10 depending on the methods of the MgO introduction. This improvement becomes more dramatic at higher fields and reaches the maximum, the highest temperature studied, where the Bi-2212 tapes with deposited $\mathrm{MgO}$, as well as tapes prepared by solvent methods and tapes prepared by settlement have about 3, 6 and 40 times higher $J_{\mathrm{c}}$ values, respectively, compared to the results achieved for the tapes without the $\mathrm{MgO}$ addition.

The above results indicate that the uniformity of the MgO nanoparticle distribution enhances the transport properties of $\mathrm{Bi}-2212 / \mathrm{Ag}$ tapes and individual particles act as effective flux-pinning centers. The highest improvement of flux pinning potential occurs in a certain region of temperature and magnetic field. Similar behavior was observed for $\mathrm{Bi}-2212$ tapes with 200nm MgO particles [4], where the largest enhancement was achieved at $15 \mathrm{~K}$. 


\section{Conclusions}

Bi-2212/Ag tapes with and without nanosize MgO additions were prepared by four different methods. The microstructure and superconducting properties of the partially melted tapes have been investigated. MgO particles dispersed in the $\mathrm{Bi}-2212$ matrix retain cubic morphology and no reaction with the $\mathrm{Bi}-2212$ matrix was observed. The distribution of MgO nanoparticles in the Bi-2212 matrix depends on the methods of tape preparation. The most uniform distribution was achieved for Bi2212 tapes prepared by the settlement method.

It was found that a significant enhancement in magnetization hysteresis can be achieved in the tapes produced by all four described methods of MgO embedment. However, the best results were obtained for the tapes prepared by the settlement method, indicating that the uniformity of the flux pinning centers distribution plays a significant role in the tapes performance in the applied magnetic field.

Within the investigated temperature and magnetic field ranges, the largest improvement of $J_{\mathrm{c}}$ over the un-doped $\mathrm{Bi}-2212$ tapes was achieved at the highest temperature and magnetic field studied.

\section{Acknowledgments}

The authors would like to thank Dr Toru Yamashita for useful discussion of the results. Technical support of the Korea Basic Science Institute for magnetization measurements is gratefully acknowledged. 


\section{References}

[1] J. Kase, N. Irisawa, T. Morimoto, K. Togano, H. Kumakura, D.R. Dietderich, H. Maeda, Appl. Phys. Lett. 56 (1990) 970.

[2] M.S. Walker, D.W. Hazelton, M.T. Gardner, J.A. Rice, D.G. Walker, C.M. Trautwein, N.J. Ternullo, X. Shi, J.M. Weloth, R.S. Sokolowski, IEEE Trans. Appl. Supercond. 7 (1997) 889.

[3] P.E. Kazin, M. Jansen, A. Larrea, G.F. de La Fuente, Yu.D. Tretyakov, Physica C 253 (1995) 391.

[4] S. Huang, D. Dew-Hughes, D.N. Zheng, R. Jenkins, Supercond. Sci. Technol. 9 (1996) 368.

[5] N. Adamopoulos, B. Soylu, Y. Yan, J.E. Evetts, Physica C 242 (1995) 68.

[6] S. Pavard, C. Villard, D. Bourgault, R. Tournier, Supercond. Sci. Technol. 11 (1998) 1359.

[7] B. Ni, K. Asayama, S. Kiyuna, Physica C 372-376 (2002) 1868.

[8] B. Ni, Physica C 386 (2003) 300.

[9] W. Wei, Y. Sun, J. Schwartz, K. Goretta, U. Balachandran, A. Bhargava, IEEE Trans. Appl. Superconduct. 7 (1997) 1556.

[10] A. Bhargava, J.A. Alarco, I.D.R. Mackinnon, D. Page, A. Ilyushechkin, Mater. Lett. 34 (1998) 133.

[11] A. Ilyushechkin, I.E. Agranovski, I.S. Altman, N. Racha, M. Choi, Supercond. Sci. Technol. 18 (2005) 1123.

[12] I.S. Altman, I.E. Agranovski, M. Choi, Appl. Phys. Lett. 84 (2004) 5130.

[13] A.Y. Ilyushechkin, T. Yamashita, I.D.R. Mackinnon, Physica C 377 (2002) 362.

[14] D. Buhl, T. Lang, L.J. Gauckler, Supercond. Sci. Technol. 10 (1997) 32.

[15] C.P. Bean, Rev. Mod. Phys. 36 (1964) 39. 


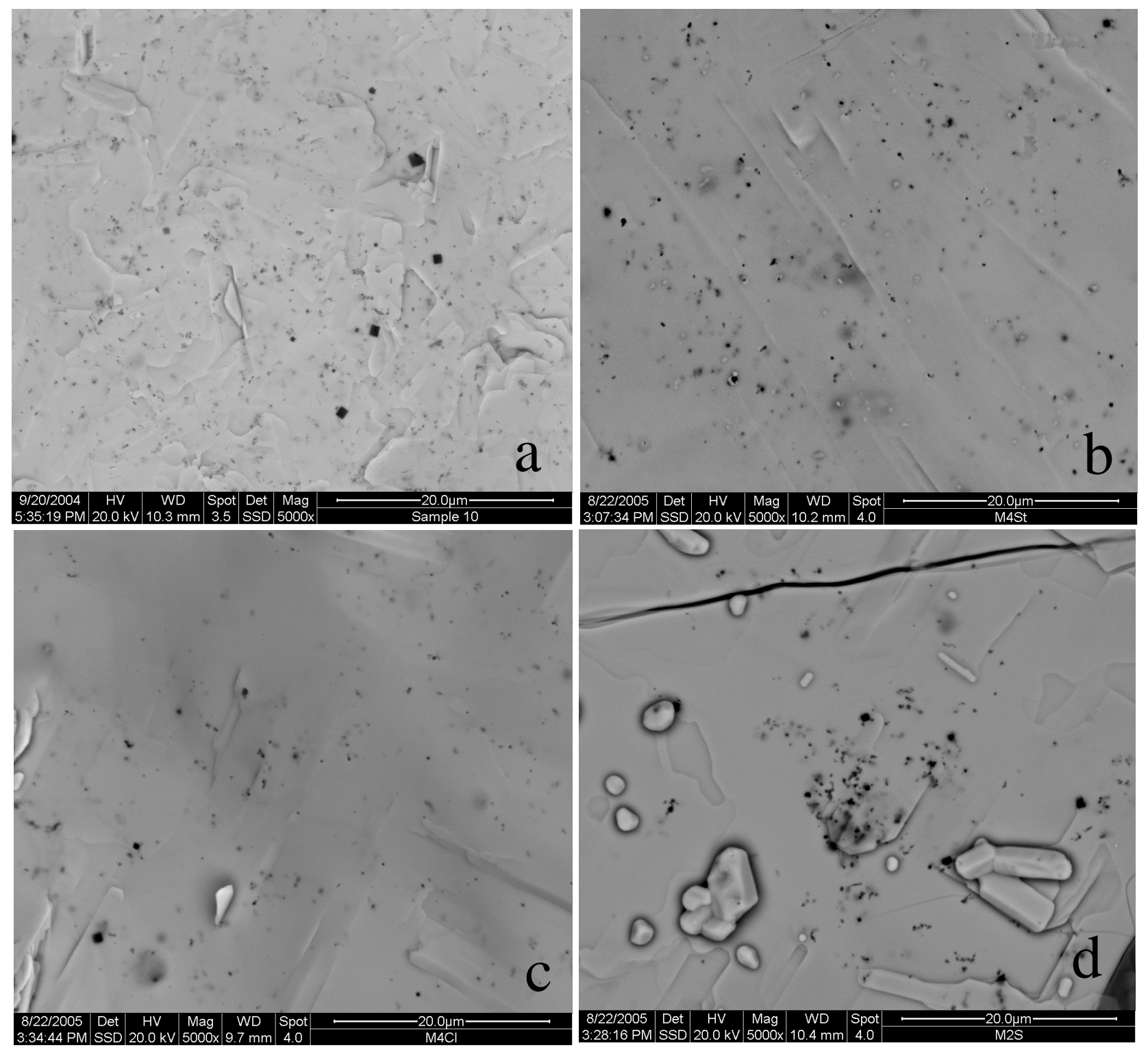

Fig. 1. SEM images of the $\mathrm{Bi}-2212+\mathrm{MgO}$ on the Ag substrate, prepared by the $\mathrm{MgO}$ deposition onto the surface of coated tape (a); settlement method (b); by MgO mixed in with solvent (c) and $\mathrm{MgO}$ deposited onto the solvent (d). 


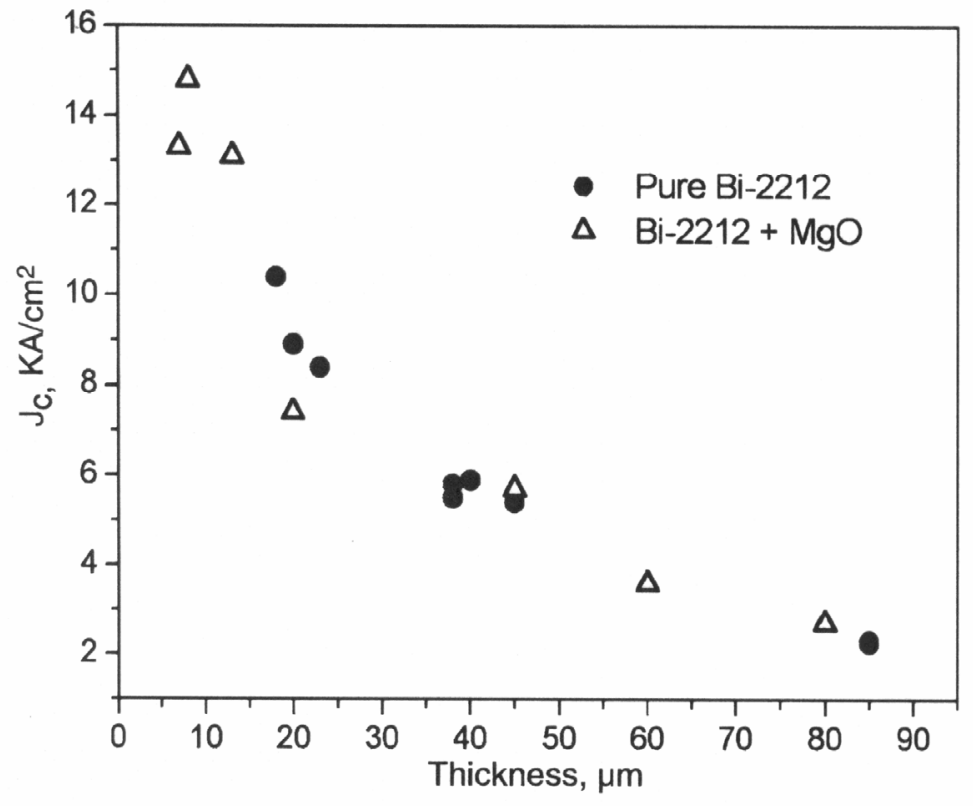

Fig. 2. Dependence of critical current densities ( $J_{c}$ 's) on tape thickness for the undoped and MgO-doped Bi-2212 tapes. 

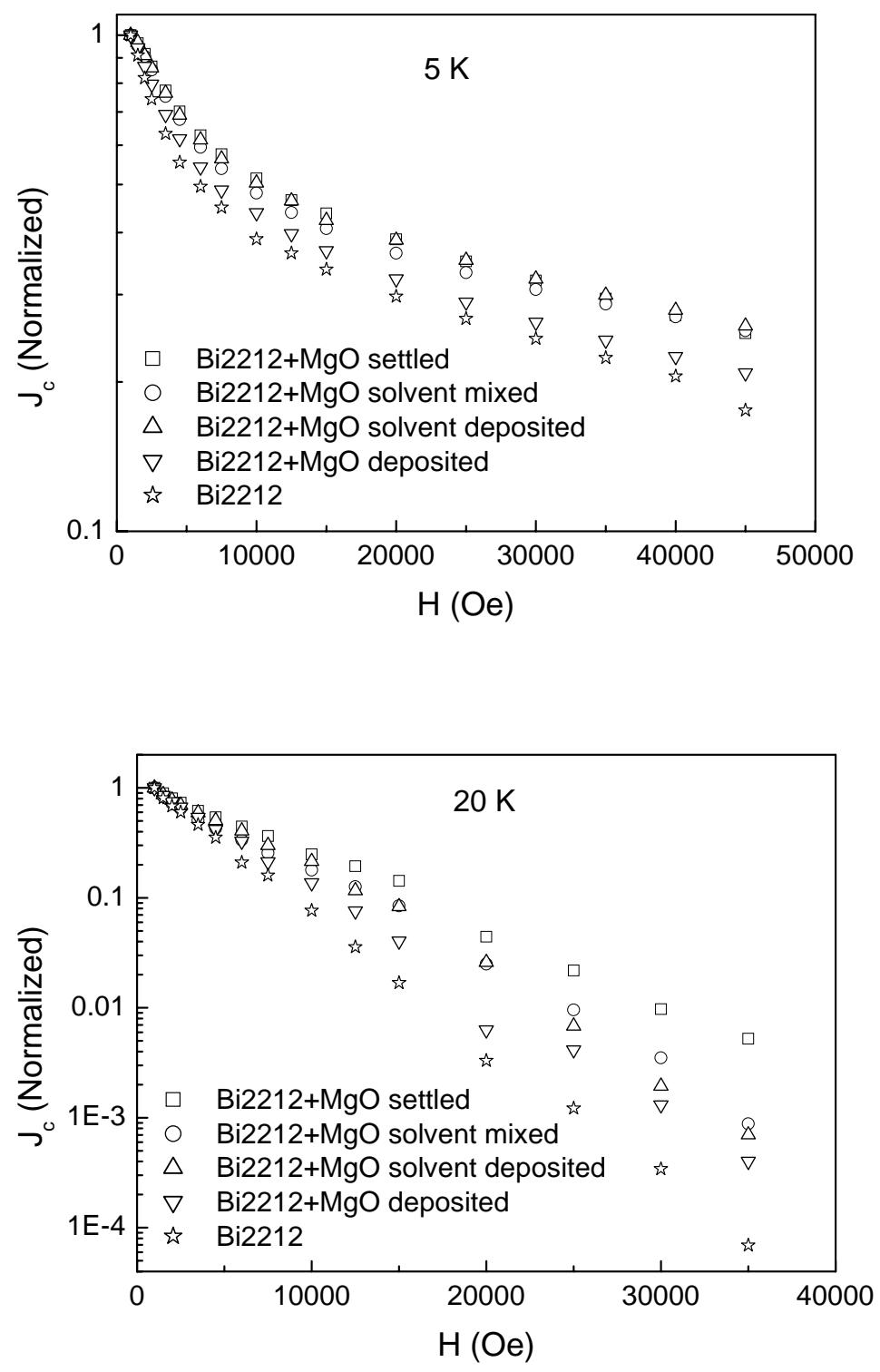

Fig. 3. Dependence of critical current densities ( $J_{\mathrm{c}}$ 's) on magnetic field $(H)$ in the un-doped and MgO-doped tapes at $5 \mathrm{~K}$ (a) and $20 \mathrm{~K}$ (b). 

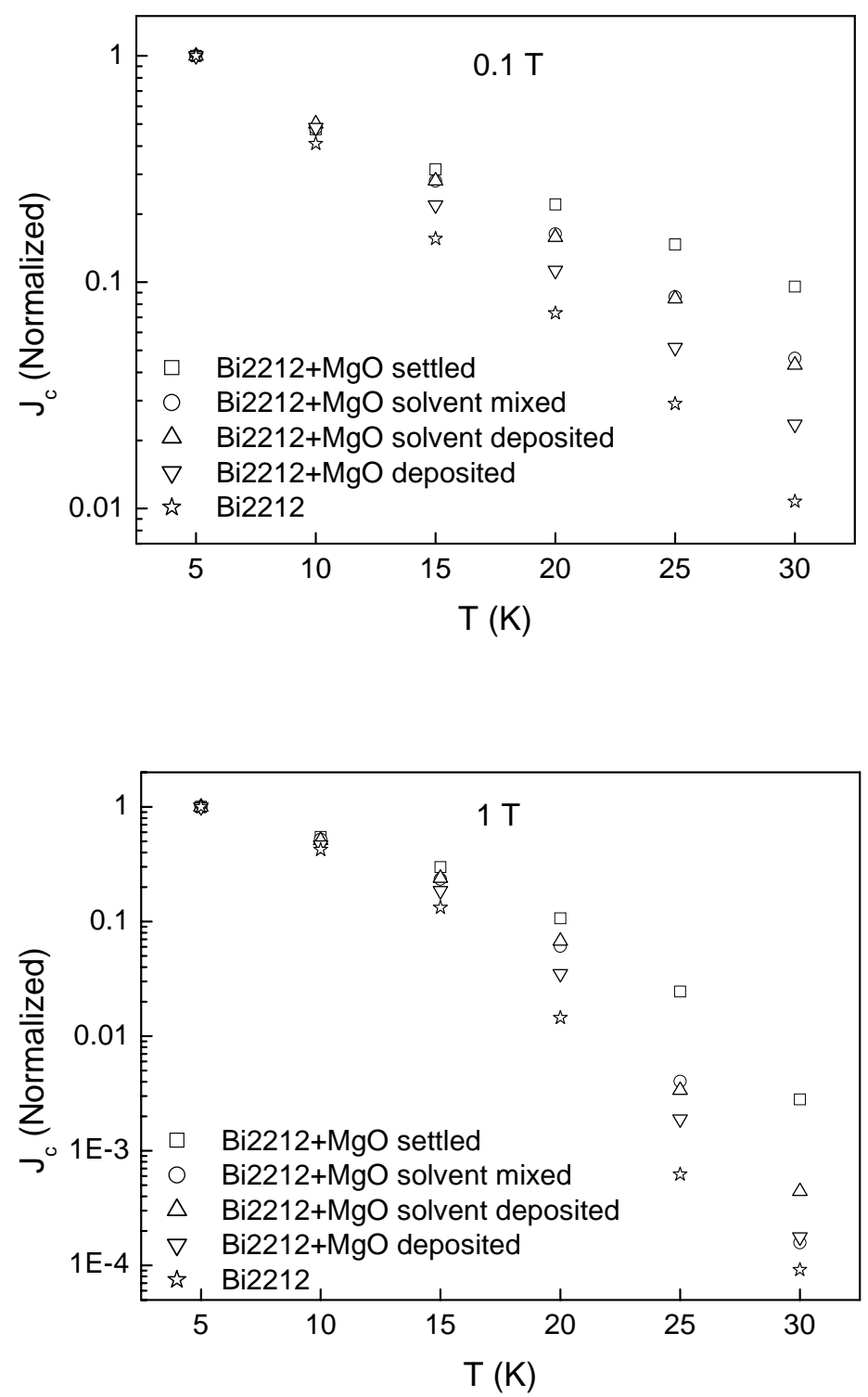

Fig. 4. Dependence of critical current densities $\left(J_{\mathrm{C}}\right.$ 's $)$ on temperature $(T)$ in the undoped and MgO-doped tapes at $H=0.1 \mathrm{~T}$ (a) and $H=1 \mathrm{~T}$ (b). 